\title{
Highly interconnected ordered mesoporous carbon - carbon nanotube nanocomposites: Pt-free, highly efficient, and durable counter electrode for dye-sensitized solar cells
}

\author{
Yimhyun Jo, Jae Yeong Cheon, Jeonghun Yu, Hu Young Jeong, Chi-Hwan Han, \\ Sang Hoon Joo*, and Yongseok Jun* \\ KIER-UNIST Advanced Center for Energy, Ulsan National Institute of Science and Technology (UNIST), \\ Ulsan689-798 (yjun@unist.ac.kr )
}

\begin{abstract}
Dye-sensitized solar cells (DSSCs) that convert solar energy into electrical energy have been of considerable interest during the last two decades because of their environmental benignity, simple fabrication process, and relatively high efficiency. Most DSSCs consist of a $\mathrm{TiO}_{2}$ working electrode with a dye adsorbed on its surface, an electrolyte with an iodine complex, and a counter electrode (CE) with a Pt coating. The $\mathrm{CE}$ is one of the most important components in the DSSCs; it returns electrons to the electrolyte via iodide reduction. Yet, thus far, only a limited number of materials have been successfully used as CEs owing to the corrosion of metals by the iodine electrolyte. Thermally platinized fluorine-doped tin oxide (FTO) is currently the prevalent material of choice, primarily because of its high electrocatalytic activity. However, high costs and limited supplies of Pt have triggered a great deal of recent efforts toward seeking low-cost alternatives for Pt. Recently emerged nanostructured carbons such as CNTs, graphene, and OMCs have appealing structural properties that are beneficial for DSSCs. As such, these nanocarbon-based CEs have shown promising applicability for DSSCs. However, the efficiency of these DSSCs in most cases is inferior to that of DSSCs with a Pt-based CE.

Herein, we report the preparation of nanocomposites of OMC and CNTs and their successful application as novel, Ptfree, highly efficient and durable DSSC CEs. OMC-CNT nanocomposites have a unique structure wherein the primary particles of the OMC are interconnected via the CNTs, thus exhibiting a medusa-like morphology. Importantly, the OMCCNT nanocomposites combine the advantageous properties of both OMC and CNTs. The high surface area of OMC provides numerous catalytically active sites for iodine reduction while its three-dimensionally (3-D) interconnected mesoporous structure allows for the facile transports of ions and electrons. In addition, CNTs can function as electrical bridges that interconnect separated OMC primary particles, thus generating fast electrical networks. We show that the DSSC employing the OMC-CNT-based CE exhibits an excellent cell efficiency, which rivals that of the DSSC cell with a conventional Pt-based CE. Furthermore, we demonstrate that the DSSC with the OMC-CNTs-based CE shows remarkable stability in a long-term efficiency test.
\end{abstract}

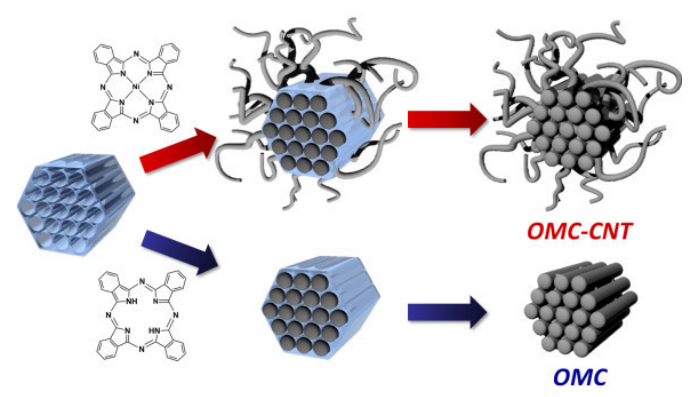

